\title{
Inserción de un implante simultáneo a la extracción de un canino superior incluido
}

\author{
FLORES-RUIZ R* \\ INFANTE-COSSÍO $\mathbf{P} * *$ \\ LLAMAS-CARRERAS JM $* * *$ \\ MARTÍNEZ-DE-FUENTES R**** \\ MAGALLANES ABAD N* \\ GUTIÉRREZ-PÉREZ JL*****
}

\begin{abstract}
Flores-Ruiz R, Infante-Cossío P, Llamas-Carreras JM, Martínez-deFuentes R, Magallanes Abad N, Gutiérrez-Pérez JL. Inserción de un implante simultáneo a la extracción de un canino superior incluido. Av Periodon Implantol. 2007; 19, 2: 85-89.
\end{abstract}

\begin{abstract}
RESUMEN
Presentamos el caso clínico de una paciente adulta con un canino superior incluido, en la que se llevó a cabo un plan de tratamiento multidisciplinar y secuencial quirúrgico-ortodóncicoprotésico. En un primer tiempo, se instauró un tratamiento ortodóncico con el que disponer de un espacio intercalar adecuado para la posterior reposición del canino superior. En un segundo tiempo, se realizó la extracción del canino incluido junto con un tratamiento combinado de regeneración ósea e inserción inmediata de un implante, para finalizar con la restauración protésica mediante una corona implantosoportada. Se revisa además la literatura al respecto y el estado actual del problema.
\end{abstract}

\section{PALABRAS CLAVE}

Canino incluido, implante simultáneo.

Fecha de recepción: Septiembre 2006.

Fecha de aceptación: Octubre 2006.

\section{INTRODUCCIÓN}

Las posibilidades terapéuticas ante un canino incluido superior son diversas. El plan de tratamiento puede incluir la extracción quirúrgica, las modalidades combinadas de tratamientos quirúrgico-ortodóncicos y el autotrasplantes. Sin embargo, el hallazgo de un canino superior incluido en un paciente adulto representa un caso especial de manejo terapéutico, ya que cuando se diagnostica a menudo han provocado pro- blemas eruptivos, mecánicos, nerviosos o quísticos asociados que justifican la extracción del canino incluido, así como una falta de espacio en el arco dentario, lo cual nos condiciona la rehabilitación protésica tras la extracción del canino ${ }^{(1)}$.

El objetivo de este artículo es presentar un caso clínico poco común de un tratamiento combinado y secuencial para solucionar un canino superior incluido en una paciente adulta mediante ortodoncia, extracción, in-

\footnotetext{
* Becario/a de Investigación. Alumno/a del Máster de Cirugía Bucal. Universidad de Sevilla

** Profesor Asociado de Cirugía Bucal. Facultad de Odontología. Universidad de Sevilla.

*** Profesor Asociado de Ortodoncia. Facultad de Odontología. Universidad de Sevilla.

**** Profesor Asociado de Prótesis Estomatológica. Facultad de Odontología. Universidad de Sevilla.

***** Profesor Titular Vinculado de Cirugía Bucal. Facultad de Odontología. Universidad de Sevilla.
} 
serción inmediata de un implante y restauración implantoprotésica. Se revisa además la literatura al respecto y el estado actual del problema.

\section{CASO CLÍNICO}

Una paciente de 50 años de edad sin antecedentes patológicos médicos de interés, acudió remitida por su odontólogo a la consulta del Servicio de Cirugía Oral y Maxilofacial del Hospital Universitario Virgen del Rocío de Sevilla, para la extracción de un canino superior izquierdo incluido. La paciente refería que hacía 6 meses se le había exfoliado el canino superior caduco espontáneamente. Tras realizar una radiografía panorámica y una radiografía lateral, se comprobó la situación del canino incluido en el cuadrante superior izquierdo, impactado entre el incisivo lateral y central, con el desarrollo radicular y del foramen apical completo (Figuras lA y 1B). A la exploración intraoral, se observó la ausencia del segundo premolar superior derecho y el primer molar inferior izquierdo, de largo tiempo de evolución. Presentaba diastemas en el sector anterior y una distancia del tramo edéntulo inferior a $5 \mathrm{~mm}$ (Figura 1C). Tras valorar las distintas opciones terapéuticas, se le planteó a la paciente realizar en primer lugar un tratamiento ortodóncico con el que disponer del espacio necesario para la reposición protésica en la región canina, y en un segundo lugar tras

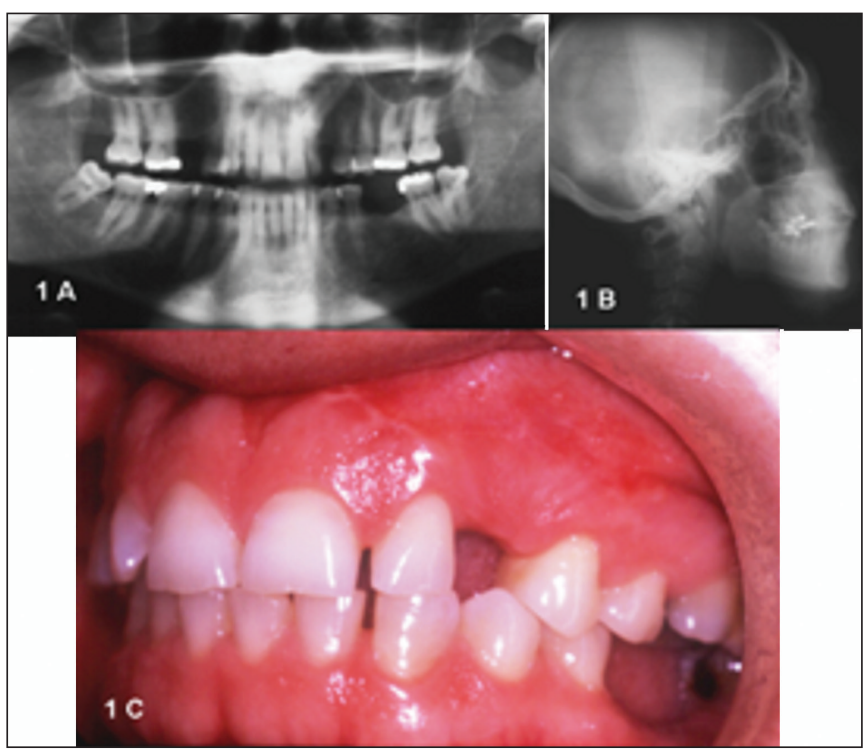

Fig. 1: A: radiografía panorámica de la paciente; $B$ : radiografía lateral; $y$ C: imagen intraoral previa tras la pérdida del canino deciduo, antes de comenzar el tratamiento ortodóncico y de la extracción del canino. la inserción de un implante osteointegrado en el mismo tiempo quirúrgico que la extracción del canino incluido proceder a la restauración protésica.

En una primera fase se llevó a cabo el tratamiento ortodóncico para conseguir un espacio mesiodistal de 7 $\mathrm{mm}$ y cierre de diastemas. Una vez finalizado éste se planificó la extracción del canino incluido y la inserción de un implante. Mediante anestesia local, se levantó un colgajo mucoperióstico por palatino para acceder al canino incluido. Tras llevar a cabo la mínima osteotomía, se realizó la odontosección del diente, su extracción y legrado del lecho (Figura 2A). A continuación se levantó un colgajo mucoperióstico vestibular para exponer la cresta y la cortical vestibular. Se colocó un implante cilíndrico de hidroxiapatita de 3,25 $\mathrm{mm}$ de diámetro y $15 \mathrm{~mm}$ de longitud, procurando el anclaje crestal hasta el suelo de las fosas nasales, consiguiendo muy buena fijación de éste. Se colocó una membrana reabsorbible de colágeno y se procedió a una regeneración ósea guiada del defecto con hueso de banco desmineralizado (Figura 2B). También en la misma sesión se insertaron de forma convencional implantes en la posición del segundo premolar superior derecho y primer molar inferior izquierdo. La evolución postoperatoria cursó sin complicaciones.

Tras un periodo de osteointegración de 6 meses, se llevó a cabo la segunda cirugía y colocación del pilar transepitelial sin incidencias (Figura $2 \mathrm{C}$ ). La recons-

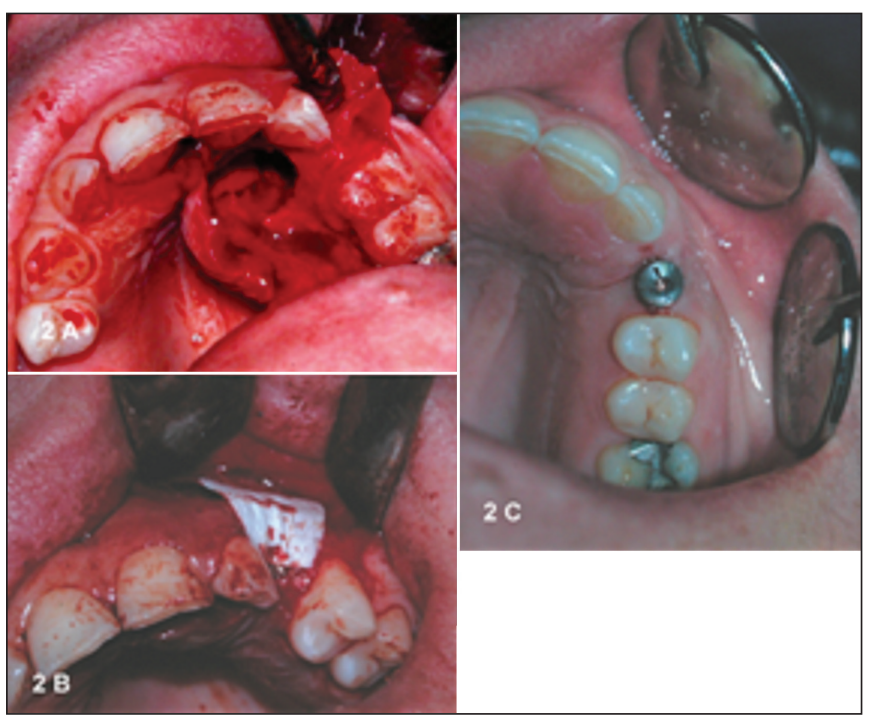

Fig.2: A: extracción del canino incluido; B: membrana reabsorbible cubriendo el implante y el material injertado; y C: imagen ocludal tras la cirugía de conexión del pilar transepitelial. 
trucción protésica se realizó con una corona implantosoportada cementada. A partir de este momento la paciente fue remitida al ortodoncista para finalizar el tratamiento de ortodoncia. Tras siete años de evolución, la evolución es favorable sin haber presentado pérdida ósea periimplantaria y con mantenimiento de la estética y funcionalidad (Figura 3).

\section{DISCUSIÓN}

La gran predictibilidad de los implantes dentales ha permitido que pueda establecerse una estrecha interrelación entre la implantología y la ortodoncia para solucionar caso complejos de ausencias dentarias. El ortodoncista puede requerir la inserción de implantes antes de iniciar el tratamiento ortodóncico para ser utilizados como unidades de anclaje que posteriormente servirán para soportar prótesis, o la inserción al finalizar los casos para reponer dientes ausentes. En otras ocasiones, la ortodoncia se indica con carácter previo a la colocación de los implantes para crear las condiciones de espacio adecuadas o para solucionar ciertos defectos óseos y de tejidos blandos que nos mejoren tanto las posibilidades de inserción de los implantes como los resultados estéticos finales.

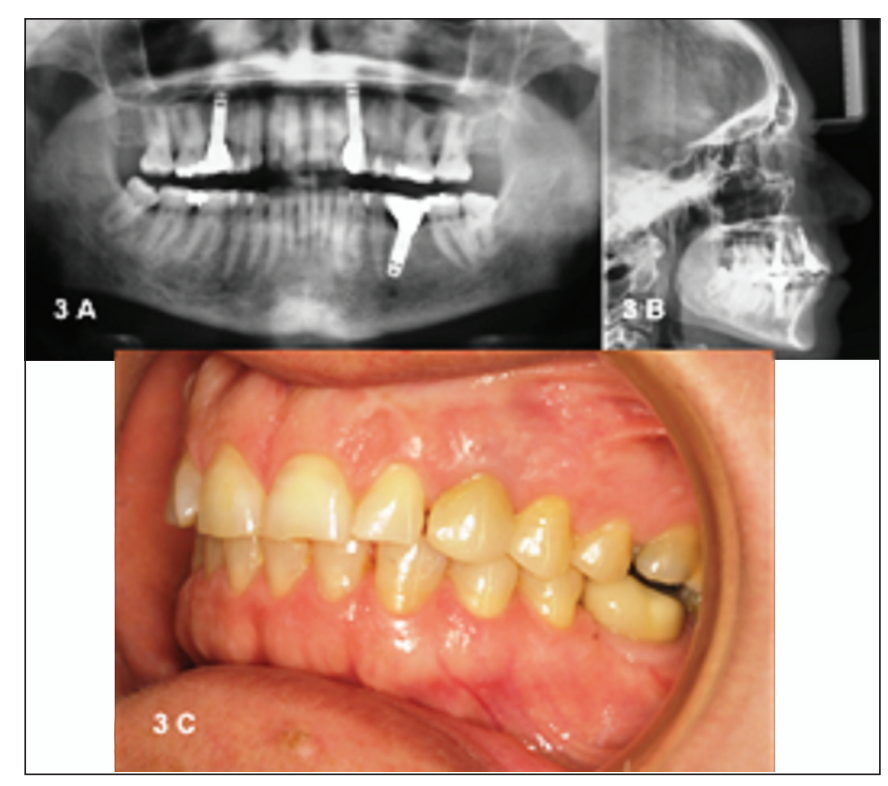

Fig.3: Control a los 7 años. A: radiografía panorámica de donde se puede observar el perfecto alineamiento de las raíces de los dientes adyacentes y el implante; $B$ : radiografía lateral; $y$ C: vista final de la restauración protésica, con mantenimiento de la estética y función.
Un caso especial se plantea en el manejo de los caninos superiores incluidos, ya que la colaboración entre ambas disciplinas puede ser una alternativa para solucionar una inclusión de un canino incluido en un paciente adulto, pues no es habitual que se empleen de forma combinada para proveer espacio suficiente e insertar un implante simultáneo a la extracción del canino incluido ${ }^{(1)}$. Es bien sabido que para la inserción de un implante para la reposición de un canino superior es necesario disponer de un espacio suficiente que permita una colocación correcta del mismo, en el sitio adecuado y dictado por la estética y por la futura función, sin crear daño en las estructuras anatómicas vecinas y en los dientes adyacentes ${ }^{(2-4)}$. Esta situación ocurre específicamente en casos de pacientes adultos con ausencia del canino tras exfoliación el canino caduco, en el que se puede haber producido una migración tanto de las coronas como de las raíces de los dientes adyacentes. Los requerimientos de espacio aconsejables que se deben conseguir mediante el tratamiento ortodóncico para la inserción de implantes deben ser unos $2 \mathrm{~mm}$ de espacio por mesial y distal desde el implante a los dientes adyacentes. Este espacio debe existir tanto a nivel coronal como radicular. Las raíces de los dientes adyacentes al espacio edéntulo deben quedar paralelas entre sí, y se deben analizar detenidamente las posibles curvas y orientaciones de las mismas. Por ello, el espacio debe ser de $7 \mathrm{~mm}$ como mínimo.

La colocación de un implante osteointegrado de forma inmediata tras la extracción de un canino incluido presenta algunas ventajas frente a la inserción diferi$\mathrm{da}^{(4-6)}$. De una parte, se acorta el período de osteointegración aprovechando el período de cicatrización tras la extracción del incluido. Permite además colocar el implante en la dirección más adecuada, escogiendo un implante de máxima longitud. En el caso presentado, empleamos un implante de $15 \mathrm{~mm}$, que fue colocado con bicorticalización en la cresta ósea y en el suelo de la fosa nasal con el fin de lograr la mayor estabilidad del implante, máxime en este caso en el que utilizamos un implante cilíndrico ${ }^{(7)}$. Desde el punto de vista quirúrgico hay que llevar a cabo una extracción lo menos traumática posible, evitando osteotomías amplias, siendo de elección el abordaje por palatino. Es necesario además levantar un colgajo vestibular para visualizar y controlar las corticales vestibular y palatina en el momento de insertar el implante. Conviene plantear la extracción mediante odontosección y proceder a legrar cuidadosamente el lecho para asegurar que se elimina todo el tejido blando remanente, como así se hizo en el caso presentado. 
La inserción de implantes inmediatos tras una exodoncia convencional es un procedimiento con tasas de éxito de más del $90 \%{ }^{(3,4,6,8,9)}$. Habitualmente este procedimiento lleva consigo el uso de hueso autólogo recogido de filtro de hueso y/o hueso de banco desmineralizado, para ser alojado en la discrepancia entre el defecto alveolar y el diámetro del implante. Este enfoque cobra mayor importancia en la extracción de dientes incluidos que llevan asociada una ostectomía mayor, requerirá mayor cantidad de hueso y por ende se preconiza el uso de una membrana que impida la invaginación de tejido epitelial en el defecto óseo ${ }^{(5-7)}$. En el caso presentado, se indicó el tratamiento ortodóncico previo a la exodoncia del canino incluido para conseguir espacio adecuado y tras la extracción del canino se procuró el relleno de la cavidad de la osteotomía, la regeneración con una membrana y el recubrimiento mucoso adecuado. Con ello se evitó el espacio muerto entre el implante y la cavidad de osteotomía que comprometiera la osteointegración.

Ante la ausencia de un diente en el sector anterior de maxilar superior las alternativas terapéuticas incluyen la rehabilitación protésica mediante prótesis removible, puente fijo convencional, prótesis parcial fija tipo Maryland o prótesis sobre implantes. Sin embargo, una de las situaciones más difíciles y complejas de rehabilitar con implantes es el sector anterior, pues se ha de colocar el implante en la situación ideal en función de las características biomecánicas y oclusales del canino superior. Pero también se ha de asegurar la estética de la restauración final consiguiendo unos tejidos blandos que simulen la papila interdentaria. Las ventajas de la rehabilitación de un canino con una corona unitaria sobre implante frente al resto de prótesis fija tradicional incluyen la conservación de la estructura dentaria de los dientes adyacentes, el máximo respeto pulpar y periodontal, la posibilidad de conseguir mayor estética y mantener el patrón oclusal del canino.

El seguimiento clínico durante 7 años y los resultados obtenidos en nuestro paciente han sido óptimos. No obstante, en la actualidad, como consecuencia de la aparición de nuevos diseños en la morfología de los implantes roscados y de tratamientos específicos sobre la superficie de los mismos, se están utilizando con gran predictibilidad procedimientos de carga inmediata que pueden suponer una alternativa a la secuencia del tratamiento como el caso que se ha presentado en este trabajo. En este sentido, se podría haber realizado la extracción del canino incluido, la regeneración ósea y la colocación de una prótesis provisional que el ortodoncista pudiera modificar durante la fase de tratamiento ortodóncico, y a los 2-4 meses insertar un implante y colocar una corona implantosoportada en el mismo día.

\section{CONCLUSIONES}

El enfoque multidisciplinar de este caso, planificando un tratamiento ortodóncico previo y un tratamiento combinado de regeneración ósea junto con la inserción de un implante tras la exodoncia de un canino incluido, nos permite una reducción del tiempo de tratamiento y plantear otra alternativa al tratamiento de prótesis fija convencional.

La inserción inmediata de implantes postextracción dentaria es una técnica en auge, la cual se puede desarrollar en los casos favorables teniendo la misma tasa de éxito que los implantes diferidos.

\section{AGRADECIMIENTOS}

\section{Al técnico en prótesis dental D. Salvador González Guerrero.}

\section{ABSTRACT}

We present a clinical case of an adult patient with an impacted upper canine, a multidisciplinary and sequential surgical-orthodontist-prosthetic treatment plan was made. In a first time, an orthodontic treatment was established in order to have a suitable space to a later upper canine replacement. In a second instance, the extraction of the canine was done, including a combined treatment of bone regeneration, with an immediate insertion of an implant. To conclude the treatment a prosthetic restoration with a crown supported on an implant was completed. Review of the literature was done in order to present the actual situation of the problem.

\section{KEY WORDS}

Included canine, Simultaneous implant.

\section{BIBLIOGRAFÍA}

1. Magheri P, Cambi S, Grandini R. Restorative alternatives for the treatment of an impacted canine: surgical and 
prosthetic considerations. Pract Proced Aesthet Dent. 2002; 14:659-64; quiz 666.

2. Mazor Z, Peleg M, Redlich M. Immediate placement of implants extraction sites of maxillary impactes canines. JADA 1999;130:1767-70.

3. Schwartz-Arad D, Chaushu G. The ways and wherefores of inmediate placement of implants into fres extraction sites: a literature review. J Periodontol 1997;68:915-23.

4. Block MS, Kent JN. Placement of endosseous implants into tooth extraction sites. J Oral Maxillofac Surg 1991; 49:1269-76.

5. Gher ME, Quitero G, Assad D, Monaco E, Richarson AE. Bone grafting anf guided bone regeneration for immediate implants in humans. J Periodontol 1994;65:881-91.

6. Augthum M, Yildirim M, Spiekermann H, Biesterfeld S. Healing of bone defects in combination with inmediate implants using the membrane tecnique. Int J Oral Maxillofac Implants 1995; 10:421-8.
7. Kent JN, Block MS. Simultaneous maxillary sinus floor bone grafting and placement of hydroxylapatitecoated implants. J Oral Maxillofac Surg 1989;47:23842.

8. Adell R, Lekholm U, Rockler B, Branemark PI. A 15-year study of osseointegrated implants in the treatment of the edentulous jaw. Int J Oral Surg 1981;10:387-416.

9. Van Steenberghe D, Lekholm U, Bolender C, et al. Applicability of osseointegrated oral implants in the rehabilitation of partial edentulism: a prospective multicenter study on 558 fixtures. Int J Oral Maxillofac Implants 1990;5:272-81.

\section{CORRESPONDENCIA}

Rafael Flores Ruiz

Av. Kansas City 92, $8^{\circ} \mathrm{B}$

41007 SEVILLA

Correo electrónico: rafaelfloresruiz@yahoo.es 
